ARTICLE OPEN

Check for updates

\title{
REGy regulates circadian clock by modulating BMAL1 protein stability
}

\author{
Syeda Kubra $\mathbb{D}^{1,6}$, Haiyang Zhang ${ }^{1,6}$, Youwen $\mathrm{Si}^{2}$, Xiao Gao ${ }^{3}$, Tianzhen Wang ${ }^{1}$, Linian Pan ${ }^{1}$, Lei $\mathrm{Li}^{1}$, Nanzhe $\mathrm{Zhong}^{3}{ }^{{ }^{凶}}$,

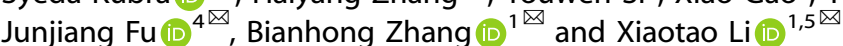

(c) The Author(s) 2021

Endogenous clocks generate rhythms in gene expression, which facilitates the organisms to cope through periodic environmental variations in accordance with 24-h light/dark time. A core question that needs to be elucidated is how such rhythms proliferate throughout the cells and regulate the dynamic physiology. In this study, we demonstrate the role of REG $\gamma$ as a new regulator of circadian clock in mice, primary MEF, and SY5Y cells. Assessment of circadian conduct reveals a difference in circadian period, wheel mode, and the ability to acclimate the external light stimulus between WT and KO littermates. Compared to WT mice, REGY KO mice attain the phase delay behavior upon light shock at early night. During the variation of 12/12 h light/dark (LD) exposure, levels of Per1, Per2, Cry1, Clock, Bmal1, and Rora circadian genes in suprachiasmatic nucleus are significantly higher in REGy KO than in WT mice, concomitant with remarkable changes in BMAL1 and PER2 proteins. In cultured cells depleted of REG $\gamma$, serum shock induces early response of the circadian genes Per1 and Per2 with the cyclic rhythm maintained. Mechanistic study indicates that REGY directly degrades BMAL1 by the non-canonical proteasome pathway independent of ATP and ubiquitin. Silencing BMAL1 abrogates the changes in circadian genes in REG $\gamma$-deficient cells. However, inhibition of GSK-3 $\beta$, a known promoter for degradation of BMAL1, exacerbates the action of REGy depletion. In conclusion, our findings define REG $\gamma$ as a new factor, which functions as a rheostat of circadian rhythms to mitigate the levels of Per1 and Per2 via proteasome-dependent degradation of BMAL1.

Cell Death Discovery (2021)7:335; https://doi.org/10.1038/s41420-021-00704-9

\section{INTRODUCTION}

Earth's atmosphere has the evolutionarily fundamental characteristic of 24-h light/dark (LD) cycle that effectuate the predominant influence on sleep-wake and activity of organisms. To coexist with the ambient variables such as light, dark, and temperature, organisms are created with a system of circadian clocks that work throughout the body, thus maintaining the life cycle upon changes in climate [1]. The mammalian circadian time keeping framework is primarily composed of the core circadian clock, the suprachiasmatic nucleus (SCN), a tiny pair of neuron clusters at the anterior hypothalamus and the subsidiary clocks in the most peripheral tissues [2]. SCN neurons instinctively operate the light signals received by the retina of eye and program the endogenous rhythms of body temperature, hormone secretion, feeding fasting, and locomotor behavior accordingly with external nature [3]. LD shifts are the major signals to orchestrate the SCN pacemaker, which subsequently work in coordination with peripheral clocks [4]. Exposure to transient light at night causes immediate changes in the circadian clock. It is predicted in the previous literature that light induces multiple signaling pathways to cause Per1 and Per2 gene expression and thus the phase changes in circadian rhythms [5]. Genetic perturbations of clock genes or environmental disturbances to circadian rhythms spark off various pathologies including sleep disorder, diabetes and cancer, suggesting that proper maintenance of circadian control is pivotal for vigorous health $[6,7]$.

At the molecular level, a core set of genes in cells in the way of transcriptional and post-translational feedback loops creates the molecular corpuscular circadian clocks, which coordinates with the metabolic cycles [8, 9]. The basic constituents of these molecular clocks include transcription factors CLOCK and BMAL1, which together forms heterodimers and binds through their PAS domain to the E-box elements of Period (Per1, Per2, Per3) and Cryptochrome (Cry1, Cry2) genes at the promoter regions. Induction of $\mathrm{Per}$ and $\mathrm{Cry}$ transcriptions ensures the performance of the positive core of the circadian clock. PER and CRY proteins translocate in the form of PER-CRY complex to the nucleus to obstruct the CLOCK-BMAL1-mediated transcriptional activity making the negative core of circadian clock. Therefore, the coordination functioning of these genes generates the $\sim 24-\mathrm{h}$ circadian biorhythms [10]. Orphan nuclear receptors REV-ERBa, REV-ERB $\beta$, and RORa proteins work in another interconnecting

\footnotetext{
${ }^{1}$ Shanghai Key Laboratory of Regulatory Biology, Institute of Biomedical Sciences, School of Life Sciences, East China Normal University, 500 Dongchuan Road, 200241 Shanghai, China. ${ }^{2}$ Ministry of Education, Shanghai Key Laboratory of Brain Functional Genomics, East China Normal University, Shanghai, China. ${ }^{3}$ Department of Orthopedic Oncology and Spine Tumor Center, Changzheng Hospital, Naval Medical University, 415 Fengyang Road, 200003 Shanghai, China. ${ }^{4}$ Key Laboratory of Epigenetics and Oncology, The Research Center for Preclinical Medicine, Southwest Medical University, 646000 Luzhou, Sichuan, China. ${ }^{5}$ Department of Molecular and Cellular Biology, Dan L. Duncan Cancer Center, Baylor College of Medicine, One Baylor Plaza, Houston, TX 77030, USA. ${ }^{6}$ These authors contributed equally: Syeda Kubra, Haiyang Zhang. ${ }^{\circledR}$ email: nanzhezhong@outlook.com; fujunjiang@swmu.edu.cn; bhzhang@bio.ecnu.edu.cn; xiaotaol@bcm.edu
}

Received: 29 June 2021 Revised: 17 September 2021 Accepted: 8 October 2021

Published online: 05 November 2021 
feedback loop with CLOCK-BMAL1 and contribute to the transcriptional control of the Bmal1 and Clock genes [11].

Circadian clock proteins undergo post-translational modifications (PTMs) to augment important pace in the molecular oscillations of circadian clocks. Post-translational processing of clock proteins is crucial to regulate significant biological functions such as intercellular localization of clock molecules and precise time keeping between active complex formation and repression of Per and Cry transcription. The circadian activator BMAL1 protein undergoes multitude of PTMs, including acetylation [12], phosphorylation and protein instability by kinases [13], sumoylation [14], and ubiquitylation [15]. BMAL1 is a unique core clock regulator and defects in BMAL1 leads to circadian disruption [16] and various abnormalities, such as defects in glucose-lipid metabolism [17, 18] early aging [19], skeletal mandibular hypoplasia [20], and cancer [21].

REG $\gamma$ or PA28Y (encoded by PSME3 gene) is a $28-\mathrm{kDa} 11 \mathrm{~S}$ proteasome activator. It was first discovered as a major autoantigen in the blood serum of patients with systemic lupus erythematosus [22]. REG $\gamma$ has the intrinsic property that it binds and activates the proteasome to promote the ubiquitin- and ATP-independent cleavage of intact proteins $[23,24]$, mediating a degradation pathway distinguishable from the canonical ubiquitin-proteasome system [25]. Several important studies suggest that REGy have important functions in cancer, immunity, and other pathophysiological processes [26-28]. However, the role of REG $\mathrm{H}$-20S proteasome in circadian rhythms has not been investigated.

In the current work, we have identified REGy as a new regulator of circadian rhythms by downregulating the circadian genes via proteasome-dependent degradation of BMAL1. To illuminate the role of REGY in circadian clocks, we used REGy wild-type (WT) and knockout (KO) mice as well as REG $\gamma$-deficient mouse embryonic fibroblast (MEF), and SY5Y cells. REGY KO mice achieved the phase delay behavior of running wheel upon light stimulus in constant dark/dark (D/D); however, the REG WT mice did not attain the circadian phase change phenomenon of wheel activity. We also found that REGY KO mice had short free running period than the REGy WT counterpart did. Analysis of circadian gene expression levels displayed that REGy ablation promoted transcription of a number of key circadian clock genes including Per1, Per2, Cry1, Clock, Bmal1, and Rora in REGy KO SCN and in REGY KO MEF and SY5Y ShR cells. Western blot analysis also indicated increased levels of BMAL1 and PER2 proteins in REGY-deficient MEF and SY5Y cells, suggesting that REGY impede the circadian genes and protein expression. Mechanistically, we found the degradation of BMAL1 by the REGy-proteasome system in vivo and in vitro. Given the role of glycogen synthase kinase 3beta (GSK-33), a ubiquitous serinethreonine kinase, in control of BMAL1 stability [13], inhibition of GSK-3 $\beta$ results in shortening of the circadian period in mammalian cells $[29,30]$. Consistently, we found that downregulation of REG $\gamma^{-}$ GSK3 $\beta$ pathway increased the BMAL1 levels and elevated the circadian expression of circadian clock genes in MEF and SY5Y cells. Hence, we established a potential role of REGY in the regulation of circadian clocks by specifically promoting direct proteasomal degradation of BMAL1, thereby, altering the circadian gene expression. Our results suggest a novel mechanism of REGy-proteasome system that is crucial for modulation of circadian rhythms.

\section{RESULTS \\ Mice with REGy deficiency exhibit circadian phase change upon light stimulus}

Circadian rhythms of physiology, behavior, and biochemical reactions are strongly synchronized by the endogenous circadian clocks. Wheel running activity is a relevant factor that alters the circadian rhythm of sleep-wake and feeding-fasting behavior of mice. Therefore, to study the association of REGy with circadian clocks, we evaluated wheel running behavior of the mice. When acclimated to running wheels for 1 week in 12/12 h LD followed by 3 weeks of complete DD exposure, both WT and REG $\mathrm{KO}$ littermates exhibited free running rhythms in DD (Fig. 1A, B). Compared to WT mice, REG $\mathrm{KO}$ mice displayed short circadian period with less intermittent running wheel behavior (Fig. 1A, B). It is previously reported that transient light exposure causes resetting of the internal clocks and thus the locomotor activity of the animal [31]; thus we conferred the mice with a light pulse for $15 \mathrm{~min}$ at CT15 in the early night on day 8 of consecutive DD and then turned back to DD upon the completion of light pulse. While the light-induced circadian phase change had only minor effect in REG W WT mice by actogram analysis (Figs. 1C and $\mathrm{S} 1 \mathrm{~A})$, light pulse induced a significant circadian phase delay in the onset of running wheel activity in REGY KO mice (Figs. 1D and S1B). The intrinsic periods were calculated based on the data reflecting wheel activity of the mice during the DD cycle. Intrinsic period (free running natural circadian period) of REG $\mathrm{KO}$ mice was $\sim 23.5 \mathrm{~h}$, while REG WT mice had a slightly longer period of $23.9 \mathrm{~h}$, which was proximate to the previously published records for C57BL/6 mice [32] (Fig. 1E). The free running period of the REGY KO mice was $\sim 0.4 \mathrm{~h}$ shorter than that of REG $\mathrm{WT}$ mice. The results indicate that REG depletion contributes to the photic phase resetting of circadian behavior and modulates the circadian period in mice.

\section{REGy ablation promotes transcription of circadian clock- specific genes in SCN}

SCN is the fundamental architect of circadian clock in mammals and the clock gene expression is essentially required by SCN so as to synchronize the peripheral tissues in phase with external environmental changes, thus SCN reserves the circadian timekeeping function [33]. Therefore, we tested whether REGY deficiency may influence the expression of core circadian clock genes in mouse SCN. Real-time quantitative polymerase chain reaction (qPCR) and gel-based PCR analysis revealed that the basal mRNA expressions of circadian clock genes were significantly higher in SCN of REGy KO mice than in WT control (Fig. S2A, B). Next, having established that the circadian clock genes are significantly upregulated in the SCN of REG $\gamma$ KO mice compared to REG W WT mice SCN, we entrained WT and REG KO mice in 12/12 h LD cycle for 7 days, sacrificed the mice at $6 \mathrm{~h}$ intervals of ZT (zeitgeber time), and collected the SCN tissues within anterior hypothalamus sections. From real-time qPCR analysis, we found that, despite of the systematic increase of clock-specific genes such as Per2, Per1, Cry1, Clock, Bmal1, and Rora in REGy KO SCN, the circadian genes follow similar patterns over ZT both in REG $\gamma$ KO and WT SCN (Fig. 2). In line with previous reports, Per2 mRNA also reached highest levels around ZT14, both in WT and REGY KO SCN [34]. These observations suggest that REGY deficiency increases the expression of clock-specific genes in SCN of REGY KO mice compared to WT control.

\section{REGY deficiency upregulates the rhythm of circadian genes in primary MEF and SY5Y cell line}

To determine the influence of REGY on rhythmic expression of the circadian genes in vitro, we conducted real-time $\mathrm{qPCR}$ and gelbased PCR analysis in REGY WT/KO primary MEF and neuronal SY5Y cells with or without stable knockdown of REGY (ShN and ShR cells). Consistent with in vivo expression of circadian genes in SCN, Per1/2, Cry1, Clock, Bmal1, and Ror a were expressed higher in MEF KO (Fig. S3A, B) and SY5Y ShR cells (REGy knockdown cells) (Fig. S3C, D) compared to WT and ShN control cells. Given that the circadian oscillations are self-sustained in individual cell lines, so to validate the impact of REGY on cyclic rhythm of circadian genes, we treated MEF and SY5Y cells with $50 \%$ horse serum for $2 \mathrm{~h}$ followed by serum-free medium and then harvested the cells at $4 \mathrm{~h}$ intervals for a $24 \mathrm{~h}$ cycle. Gel-based PCR analysis revealed that the rhythmic patterns of Cry1, Clock, and Bmal1 genes in WT and REGy-deficient MEF KO cells were generally indistinguishable (Fig. S4A); however, Per2 and Per 1 mRNA were promptly induced with significant phase advance in response to serum shock in MEF 


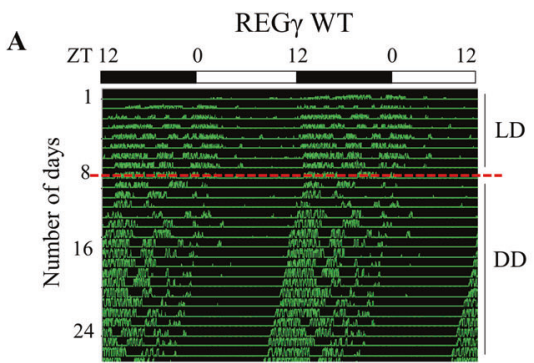

C

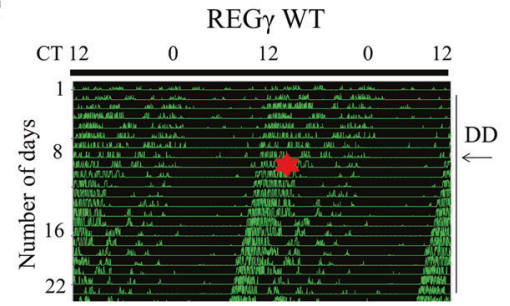

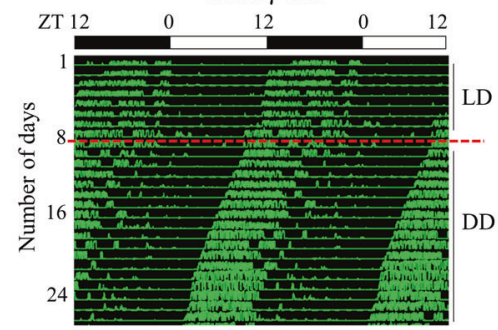

D

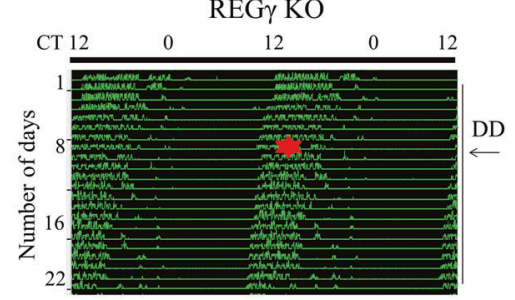

E

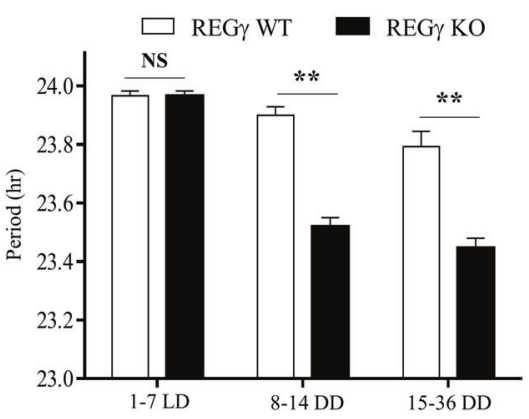

Fig. 1 Light induced circadian phase shift in REGy KO mice. A The actogram of 5-month-old REG $\gamma$ WT control mice; B the actogram of littermate REG $\gamma$ KO male mice in 12/12 h light-dark (LD) cycle followed by dark-dark (DD) for 3 weeks. For circadian phase change experiment, mice were first entrained to complete dark-dark (DD) cycle for 1 week and then placed in complete darkness for 3 weeks, and then a light pulse for 15 min at CT15 on day 8 in dark-dark (DD) was applied. REG $\gamma$ WT mice in C did not accomplish the phase delay behavior of circadian rhythm, REG $\gamma$ KO mice in D executed phase delay behavior upon light stimulus. Red dotted lines in $\mathbf{A}$, B show the start day for constant darkness. Red star and black arrows in C, D indicate the day when a light pulse was applied. E Bar graph represent intrinsic periods (natural free running periods) of mice during the first week of 12/12 h LD cycle and initial 3 weeks in constant darkness. Values in $\mathbf{E}$ represent the mean \pm SD. ${ }^{* *} p<0.01 ; t$ test, WT vs. KO. The sample size was $n=5$.

KO cells compared to MEF WT cells (Figs. 3A and S4A). Similarly, the periodic levels of Cry1, Clock, and Bmal1 transcripts in SY5Y ShR cells also had no profound differences to that in ShN cells (Fig. S4B). However, Per2 and Per1 mRNA were induced more readily in ShR cells compared to ShN cells (Figs. 3B and S4B). Consistent with changes in transcript levels, western blot results also indicated that both PER2 and BMAL1 protein expression levels were significantly elevated after serum shock in REGYdepleted primary MEF KO (Fig. 3C) and SY5Y ShR (Fig. 3D) cells compared to control cells. These findings demonstrated that REG $\gamma$ is critical for surveillance of normal circadian clock phenomenon in mammalian cells.

\section{REGy negatively regulates BMAL1 by directly promoting its proteasome-dependent degradation}

Analysis of PER2 and BMAL1 protein expression in cultured cells raised a question whether one of the key regulatory circadian proteins is targeted for degradation by the REG $\mathrm{p}$-20S proteasome pathway. Thus, we examined the overall expression of BMAL1 and PER2 proteins and we found that BMAL1 and PER2 protein were significantly high in REGY KO mice SCN and MEF KO and REGY knockdown SY5Y ShR than in WT controls (Fig. 4A). Given the role of BMAL1 as an upstream regulator of PER2, we tested whether BMAL1 is primarily targeted by the REGy-20S proteasome pathway. Thus, physical interactions between REGy and BMAL1 were analyzed in vitro, using exogenously expressed Flag-REG $y$ and HABMAL1 in 293T cells. Reciprocal co-immunoprecipitation suggested a robust interaction between the two proteins (Fig. 4B, C). We then investigated the dynamic stability of BMAL1 and PER2 proteins in the presence of cycloheximide (an inhibitor of de novo protein synthesis) for different times points in WT and REGY KO MEF and SY5Y ShN/ShR cells. BMAL1 protein was degraded with slower rate in REGY-deficient MEF and SY5Y ShR cells than in MEF WT (Fig. 4D) and SY5Y ShN control cells (Fig. 4E), which implies that REG $\gamma$ may facilitate BMAL1 degradation. However, the decay rate for PER2 protein in WT and REGy-depleted cells was comparable (Fig. 4D, E), suggesting that the negative regulation of PER2 by REG $\gamma$ may be secondary to the changes in BMAL1 protein. To confirm whether BMAL1 is a direct target of the REGY$20 \mathrm{~S}$ proteasome pathway, we performed in vitro degradation assays with purified proteins in a cell-free system. Incubation of in vitro translated BMAL1 with $20 \mathrm{~S}$ proteasome or REGy alone could not facilitate the degradation of BMAL1. However, combination of REGY and $20 \mathrm{~S}$ proteasome promoted a significant proteolysis of BMAL1 in the absence of ATP (Fig. 4F). Next, we performed a gain-of-function experiment using a previously engineered REG $\gamma$-inducible 293 cell line in which a different allele of REG $\gamma$ is expressed in the presence of doxycycline. Therefore, we carried out cycloheximide assay in doxycycline-treated cells and we found that the doxycycline-induced cells had an accelerated 

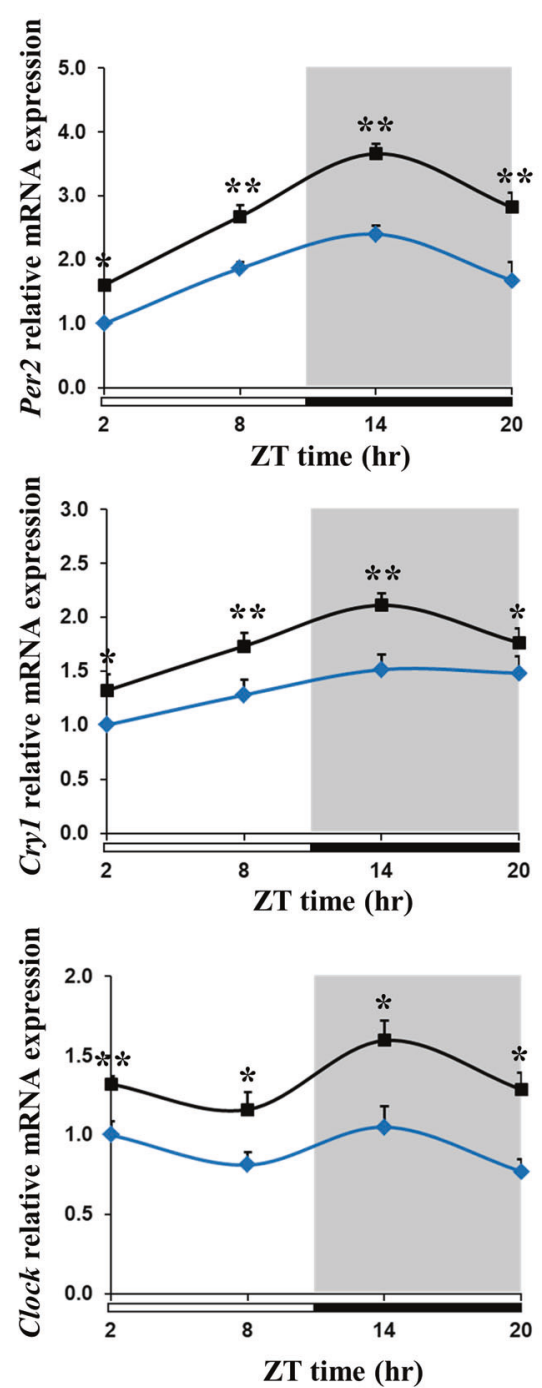
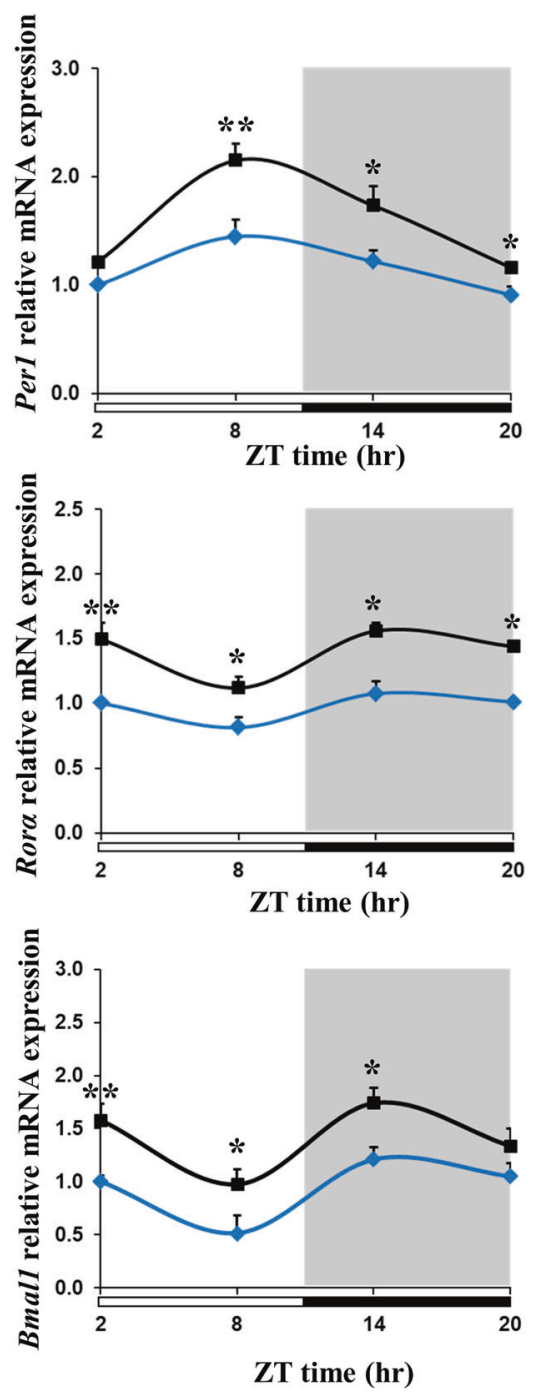

$\multimap$ SCN REG $\gamma$ WT $\rightarrow$ SCN REG $\gamma$ KO

Fig. 2 REGy deficiency promotes the expression of circadian clock-specific genes in SCN. Real-time qPCR analysis of clock-specific genes including Per1, Per2, Cry1, Clock, Bmal1, and Rora in REG $\gamma$ WT and KO mice SCN. REG $\gamma$ WT and REG $\gamma$ KO mice were entrained to 12/12 h LD cycle, then sacrificed the mice at $6 \mathrm{~h}$ intervals on the last day of the cycle and the anterior hypothalamus tissues that contain the SCN were collected for mRNA analysis. Values represent the mean $\pm \mathrm{SD}$. ${ }^{* *} p<0.01,{ }^{*} p<0.05 ; t$ test, WT vs. KO.

degradation of BMAL1 as compared to un-induced cells (Fig. 4G). 293T cells transfected with Flag-BMAL1 and HA-REGy together also showed a decrease in the expression of BMAL1 protein (Fig. 4H). Together, these findings suggest that REGY interacts directly with and promotes the degradation of BMAL1 protein, thus subsequently regulates the expression of downstream circadian genes.

\section{Alteration of circadian physiology induced by REGY abrogation is GSK-3 $\beta$-BMAL1 dependent}

Given that GSK-3 $\beta$ regulates BMAL1 protein stability and circadian function, we explored the regulation of circadian rhythms by REG $\gamma$ following manipulation of BMAL1 protein levels in primary MEF and SY5Y cells. Indeed, treatment with GSK-3 $\beta$ inhibitor (S1263) significantly increased the expression of BMAL1 and PER2 protein levels and disrupted circadian patterns in both WT and REG $Y$ KO MEF and SY5Y ShN and ShR cells compared to untreated cells (Fig. 5A, B). Similarly, upregulation of circadian genes including Per1, Per2, Cry1, Clock, and Bmal1 was observed in cells treated with $50 \%$ horse serum shock in the presence of GSK-3 $\beta$ inhibitor
(Fig. S4C, D), compared to control cells untreated with inhibitor (Fig. S4A, B). To substantiate the mechanism by which REGY regulates circadian physiology via proteasomal degradation of BMAL1, we treated SY5Y cells with the small interfering RNA (siRNA) against Bmal1 (si-Bmal1) and the control siRNA (si-Ctrl). Silencing Bmal1 markedly decreased the expression of PER2 protein both in SY5Y ShN and ShR cells, attenuating the effect of BMAL1 and PER2 upregulation induced by REGY knockdown (Fig. 5C). Consequently, ablation of Bmal1 not only blocked the effect of REGy depletion on circadian gene activation in ShR cells but also repressed overall expression of Per1, Per2, and Cry 1 genes in SY5Y ShN cells (Fig. 5D). These results indicate that REGY inhibition is a previously unknown mechanism regulating circadian biology via controlling the homeostasis of BMAL1.

\section{DISCUSSION}

Over recent years, research on REG $y$ has gained much emphasis due to its prominent functions in various biological pathways [35]. In the present work, we captivated the role of REGY in 
A $\multimap$ MEF WT $\rightarrow$ MEF KO

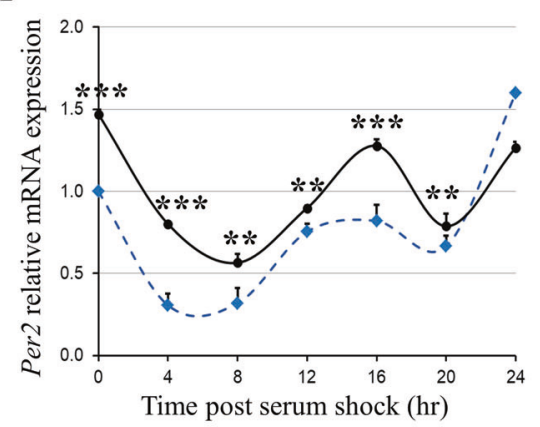

B $\quad \leftarrow$ SY SY ShN $\rightarrow-S Y 5 Y$ ShR

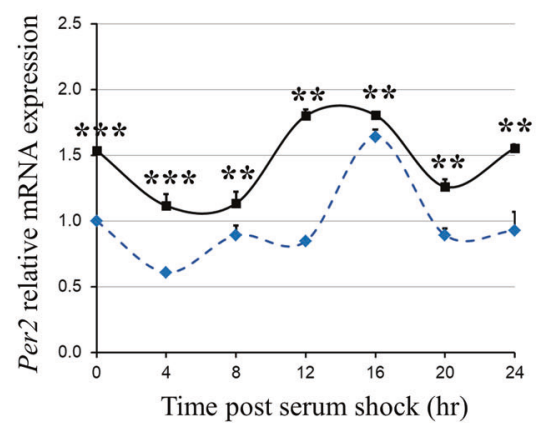

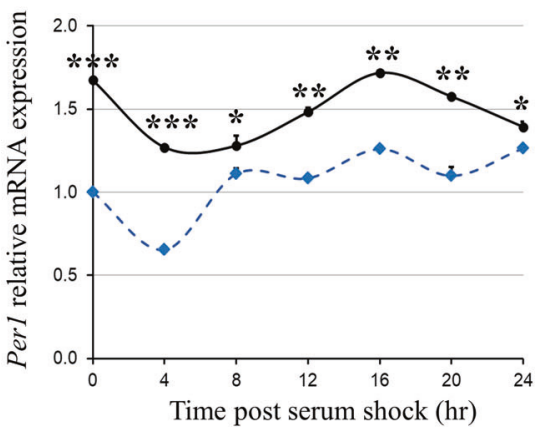

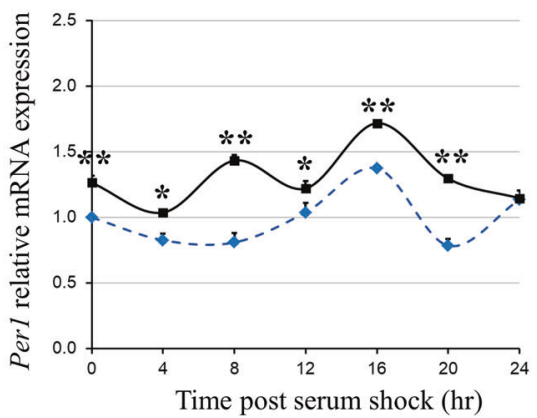

C

MEF WT

MEF KO

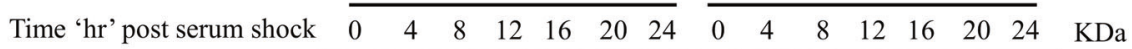

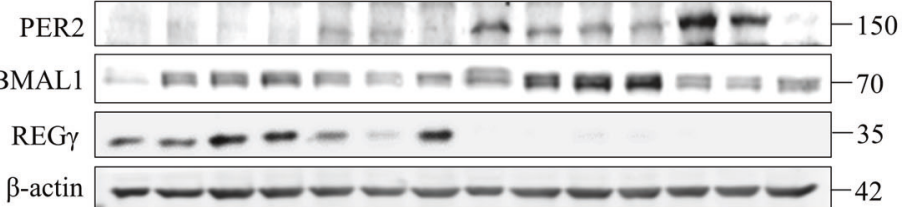

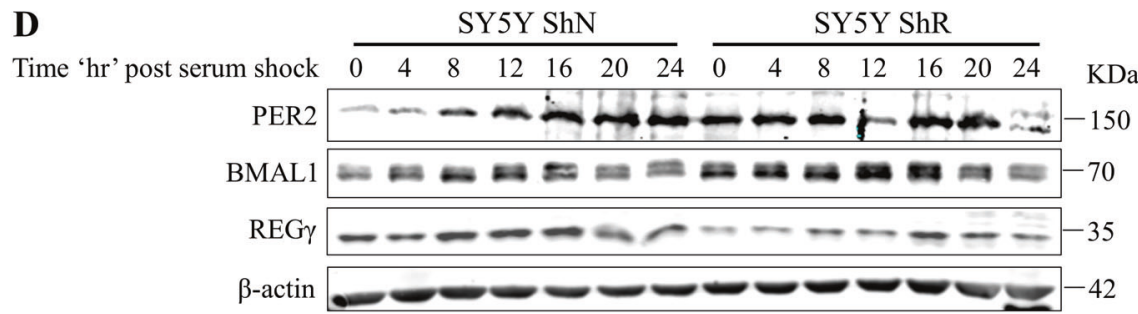

Fig. 3 REGy depletion affects the rhythms of circadian gene expression in cultured cell lines. Real-time qPCR analysis of circadian genes in A primary MEF WT/KO cells and B SY5Y ShN/ShR cells treated with $50 \%$ horse serum and then collected the cells at $4 \mathrm{~h}$ intervals across a $24-\mathrm{h}$ cycle; the target gene expression levels were analyzed relative to the ribosomal RNA gene 18S. Immunoblots of REG $\gamma$ and circadian components PER2 and BMAL1 in C MEF and D SY5Y cells. Values represent the mean \pm SD. ${ }^{* * *} p<0.001,{ }^{* *} p<0.01,{ }^{*} p<0.05 ; t$ test, WT vs. KO; ShN vs. ShR.

circadian rhythms and proposed a mechanism by which REGY impacts the expression of circadian genes in mice and in cell culture. We demonstrated that REGY as a proteasome activator can specifically promote proteasomal degradation of the circadian protein BMAL1, but not the downstream PER2. In REGy-deficient mice, the accumulation of BMAL1 accelerates the transcription of the circadian clock specific genes, such as Per1, Per2, Cry1, Clock, and Rora, which may be related with the phenotype of circadian entrainment deficiency and disrupted circadian behavior (Fig. 6). Although several experiments including in vitro (cell-free system) have confirmed the direct degradation of BMAL1 by REGY-20S proteasome. we have surprisingly found elevated level of mRNA transcript of Bmal1 in REGY KO mice SCN and in MEF KO and SY5Y ShR cells.
Since Rora was a target of CLOCK-BMAL1 and it contributes to the transcriptional activation of Bmal1 and Clock genes [36], we observed that Rora was upregulated in REG $\gamma$-depleted MEF and SY5Y cells and REGY KO SCN, which may partially explain the increase of the mRNA level of Bmal1 (Fig. 2). These results suggest a multi-layer regulation of BMAL1 by REGy. From the analysis of the intrinsic periods by monitoring free running activity on running wheels, we found that the period of WT mice in DD cycle was $23.9 \mathrm{~h}$, which was close to the values reported in other literatures $[32,37]$. In contrast, REG $\mathrm{KO}$ mice had a short period of $23.5 \mathrm{~h}$ and showed consistent wheel activity rather than intermittent wheel activity, which was similar with that observed in SIRT1 overexpression mice [38], suggesting a blocking function of REG $\gamma$ in circadian regulation. 
A

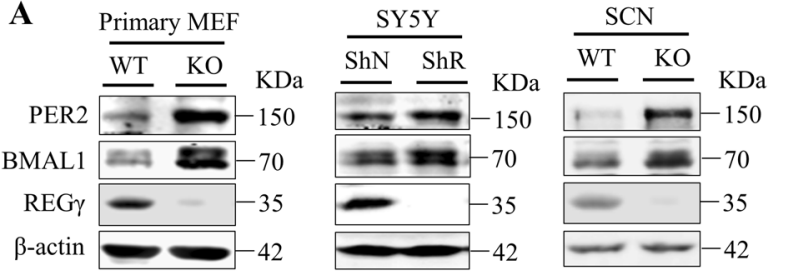

B

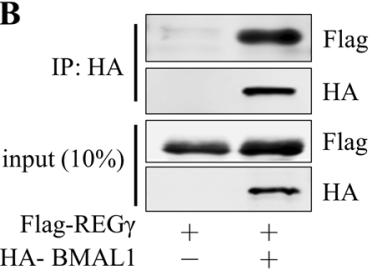

C

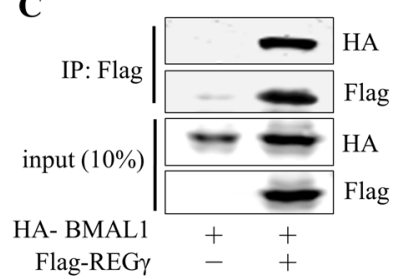

D

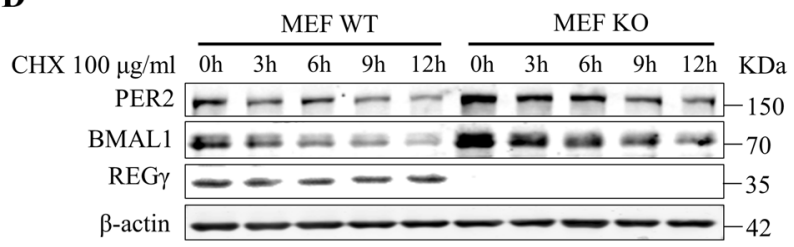

E

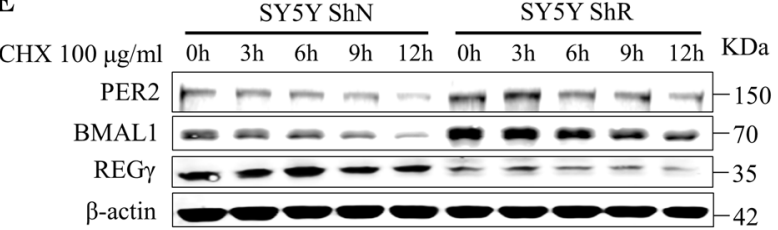

F

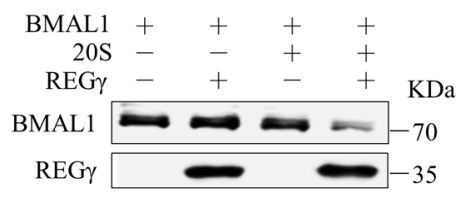

G

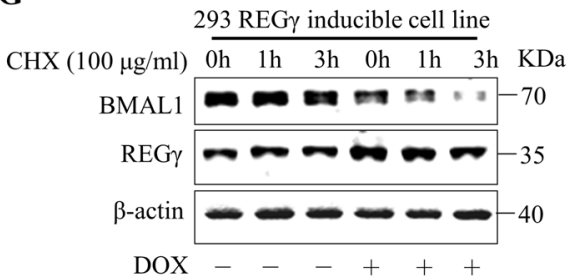

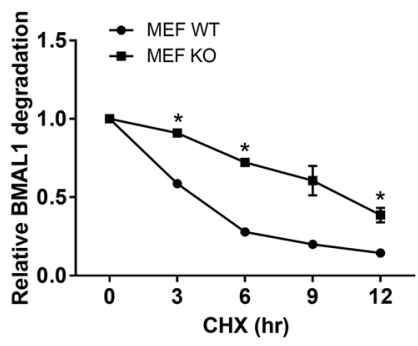

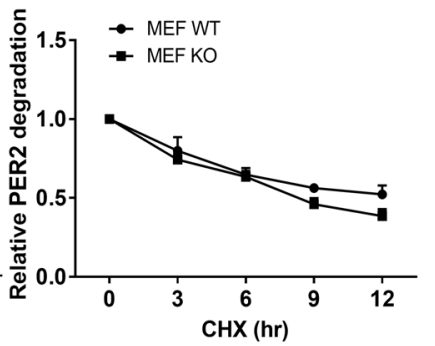

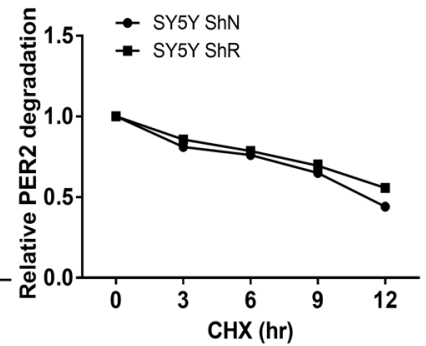

H

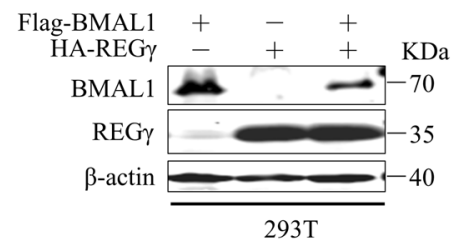

Fig. 4 REGy directly interacts with BMAL1 and promotes its degradation. A Expression of REG $\gamma$, BMAL1, and PER2 in MEF WT/KO, SY5Y ShN/ ShR cells, and REG $\gamma$ WT/KO mouse SCN. B Interaction between REG $\gamma$ and BMAL1, determined by co-immunoprecipitation and western blot analysis following transient transfection of $2 \mu \mathrm{g}$ of HA-BMAL1 and $2 \mu \mathrm{g}$ of Flag-REG $\gamma$ into 293T cells. C Reciprocal interaction between REG $\gamma$ and BMAL1 was analyzed by transient transfection of $2 \mu \mathrm{g}$ of Flag-REG $\gamma$ and $2 \mu \mathrm{g}$ of HA-BMAL1 into $293 \mathrm{~T}$ cells. D, E Stability of endogenous BMAL1 in MEFs and in SY5Y cells was analyzed in the presence of CHX $(100 \mu \mathrm{g} / \mathrm{ml})$ for the indicated time points followed by western blot analysis. $\mathbf{F}$ In vitro proteolytic analysis of REG $\gamma$-mediated degradation of BMAL1. Purified REG $\gamma$, 20S proteasome, and in vitro-translated BMAL1 were incubated at $30^{\circ} \mathrm{C}$ for $3 \mathrm{~h}$ as indicated. G Expression of REG $\gamma$ was induced by doxycycline $(1 \mu \mathrm{g} / \mathrm{ml} \mathrm{DOX}$ for $48 \mathrm{~h})$ in an engineered 293 cell line followed by western blot analysis of BMAL1 stability in the presence of cycloheximide as indicated. H 293T cells were transfected with Flag-BMAL1 and HA-REG $\gamma$ followed by western blot analysis for REG $\gamma$ and BMAL1 correlation. The quantitative results of BMAL1 stability in D, E were plotted to indicate dynamic changes. Values represent mean \pm SD. ${ }^{*} p<0.05 ; t$ test, WT vs. KO; ShN vs. ShR.

Besides the master clock SCN, the circadian clock system also exists in almost all peripheral tissues such as liver, heart, lungs, and kidneys, which maintains circadian rhythm and regulates the expression of tissue-specific genes. It has been reported that SIRT1 exhibits distinct functions and mechanisms in $\mathrm{SCN}$ and peripheral tissues [38]. Therefore, we questioned the possibilities that the variation of REGy expression over ZT in SCN or peripheral tissues could influence the expression levels of circadian genes. On the other hand, the abnormal REGy expression/function may give rise to additional circadian rhythm-associated phenotypes. It will be interesting to see whether conditional KO and/or overexpression of REGY in SCN vs. peripheral tissues may potentially lead to different phenotypes or functions via different mechanisms; all these needs to be verified by further experiments.

In recent years, more and more epidemiological and genetic data have shown that the disruption of circadian rhythms is linked to many disease-associated anomalies, such as depression, sleep disorders, cardiovascular disease, metabolic syndrome, and cancer. Metabolic rhythm disorder has been associated with multiple tumorigenesis and tumor development, including breast cancer, ovarian cancer, lung cancer, pancreatic cancer, prostate cancer, colorectal cancer, endometrial cancer, non-Hodgkin's lymphoma, osteosarcoma, leukemia, head and neck squamous cell carcinoma, and liver cancer [39]. Tumor-suppressor genes, oncogenes, and circadian clock genes can be regulated by each other. For example, loss of circadian clock genes expression lead to the increased expression of oncogene c-Myc and causes metabolic disorder. While Myc could inhibit the expression of core clock genes [27, 40], the expression of p53 is circadian, and PER2 can directly regulate the activity of p53 [41]. It is previously found that, in SCN, p53 hinders the binding of CLOCK/BMAL1 complex with PER2 promoter, resulting in inhibition of Per2 mRNA expression [42]. REGy has been reported as an oncogene and play important roles in carcinogenesis and development of many kinds of cancers [43]. Whether and how REGY and circadian clock genes reciprocally regulate each other in cancer progression deserve further exploration.

Transcriptional and post-translational events ensure the accuracy of circadian rhythms. SKP1, CULLIN1, F-box protein/ $\beta$-TrCP 
A

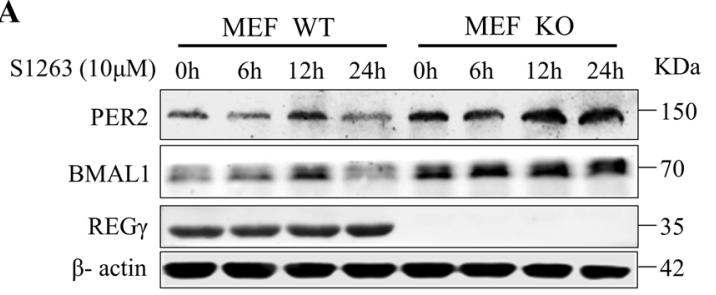

B

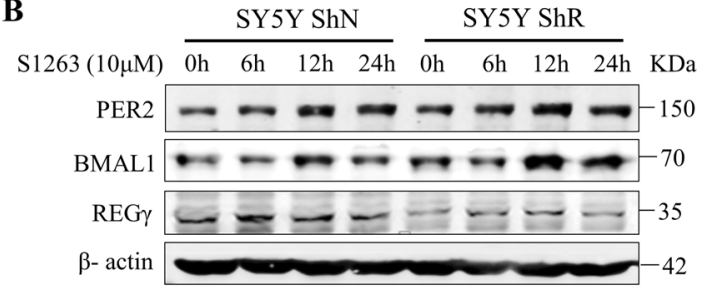

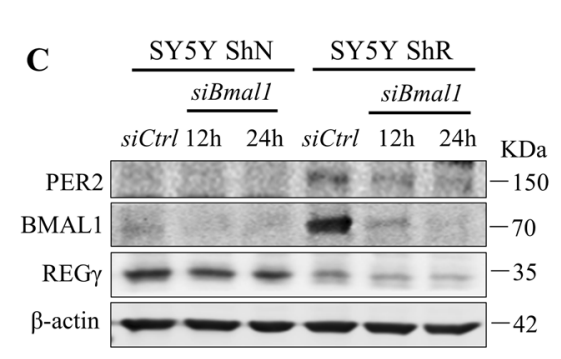
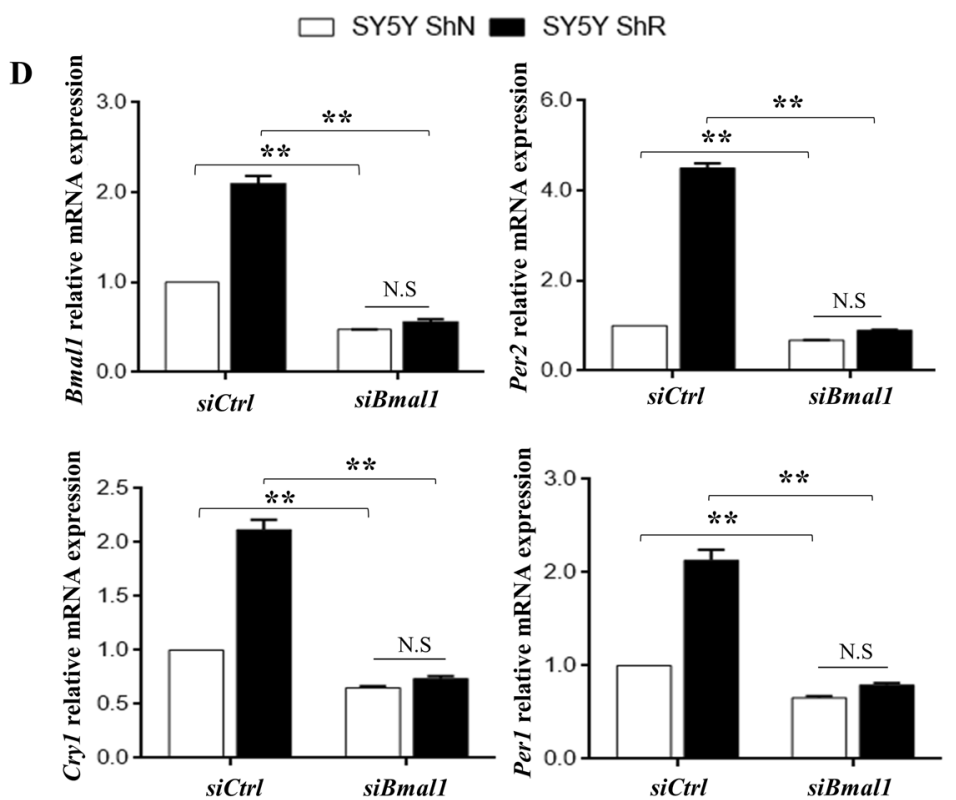

Fig. 5 Alteration of circadian physiology induced by REGy abrogation is GSK-3ß-BMAL1 dependent. A, B MEF WT/KO and SY5Y ShN/ShR cells were treated with GSK-3 $\beta$ inhibitor $(\mathrm{S} 1263,10 \mu \mathrm{M})$ for the indicated time points followed by western blot analysis. C Expression of BMAL1 and PER2 levels following BMAL1 knockdown. D The mRNA expression of circadian genes Per1, Per2, Cry1, and Bmal1 by real-time qPCR analysis in SY5Y ShN/ShR cells after the inhibition of Bmal1 by si-RNA. The data were signified as mean \pm SD. ${ }^{* *} p<0.01 ; t$ test, siBmal1 vs. control, ShN vs. ShR.

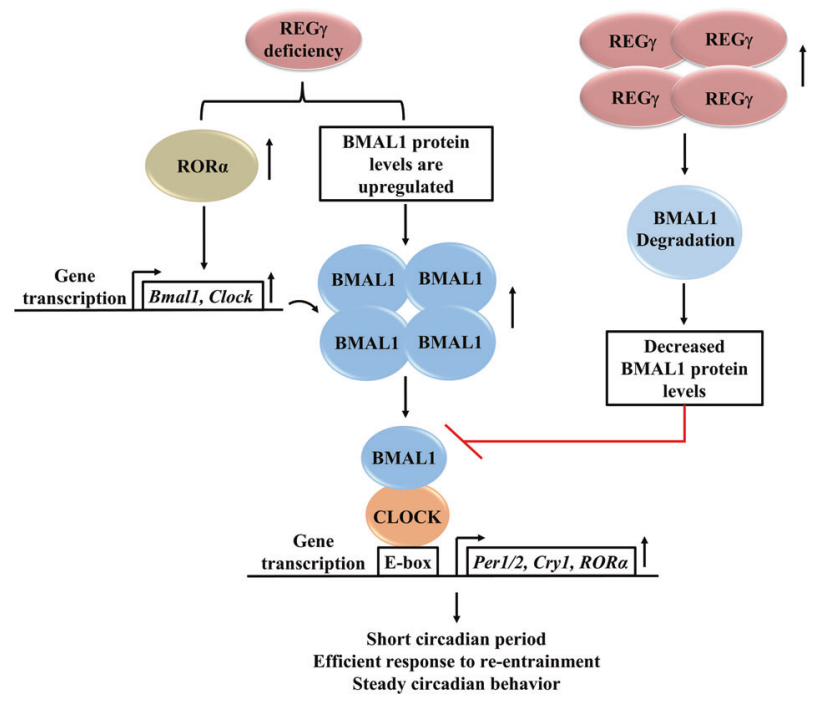

Fig. 6 A Model of REGy-dependent regulation of circadian rhythms. In mammalian cells, REG $\gamma$ promotes rapid degradation of circadian transcription factor BMAL1 to attenuate the expression of circadian core genes, thus in mice, the downregulated circadian clock causes defect in circadian entrainment and disturbed circadian rhythms. However, mice having deficiency of REG $\gamma$ display short circadian period, efficient response to re-entrainment, and steady circadian behavior. ubiquitin ligase complexes have been reported to target PER and CRY proteins for degradation [44]. BMAL1 can be modified by hybrid E2/E3 enzyme UBE2O-mediated ubiquitination to modulate its stability and associated biological functions [45]. Our findings of REGy-dependent action on circadian clock add an additional layer of regulation of the circadian system in vitro and in vivo, reinforcing the fine-tuned control of the key circadian elements. In conclusion, this study indicates that REGy-20S proteasome acts via ubiquitin- and ATP-independent pathway to promote the degradation of BMAL1, adding a new insight of the regulatory mechanism in circadian protein degradation voyage.

\section{MATERIALS AND METHODS}

Mice

In this work, REGy gene knock out mice and their WT littermate of C57BL/6 genetic inheritance were used, primitively provided by Professor John Monaco's laboratory (College of Medicine, University of Cincinnati) [46]. Mice were kept in constant temperature of $21-22^{\circ} \mathrm{C}$ and constant condition of $12 / 12 \mathrm{~h}$ LD cycle with access to food and water ad libitum. Mice were maintained according to ethical and scientific standards by "ECNU Multifunctional Platform for Innovation" (Grant Number: 011).

\section{Behavioral assay}

Wireless mouse running wheel system (ENV-047) was used for recording of mice circadian rhythms. Five-month-old mice were randomly allocated to experimental groups without blinding. For assessment of the locomotor activity rhythms, REGY KO and WT mice were housed in discretely 
ventilated running wheel cages for 2 weeks in constant 12/12 h LD sequence and then returned to complete darkness as a continuation of the dark phase of the last LD cycle. To examine the phase shift in locomotor activity, mice were exposed to light for 15 min at CT15 on day 8 in DD cycle and then returned to constant darkness for additional 8-10 days. The locomotor activities of mice and circadian phase differences were measured by the Med Associates Running Wheel Data Analysis Software. The sample size of our experiments was determined by our previous experience. The exact sample size was at least five.

\section{Cell culture and serum shock assay}

Human SY5Y cell line obtained from Cell Bank of the Chinese Academy of Sciences, Shanghai, China, was cultured in Dulbecco's modified Eagle's medium (DMEM) F12 medium. HEK 293T, primary MEF, and REGy-inducible 293 cell line were cultured in DMEM. All media were supplemented with $10 \%$ (vol $/ \mathrm{vol}$ ) fetal bovine serum (FBS) and $1 \%$ (vol/vol) $(100 \mathrm{U} / \mathrm{ml})$ HyClone penicillin-streptomycin $(P / S)$ solution. Primary MEF cells were isolated from E13.5 day of REG $\mathrm{HZ}$ mouse embryos and REGy-inducible 293 cell line was previously described [24]. Stable REG $\gamma$ knockdown SY5Y cell line was generated by integration of retroviral ShREG $\gamma$ vectors specific for REG $\gamma$ to produce ShR (ShRNA against REG $)$ ) or a control gene from OriGene (Rockville, MD) to produce ShN (ShRNA as a negative control) [47]. Cells were authenticated by a short tandem repeat profiling and routinely tested for mycoplasma contamination.

For serum shock assay, MEF and SY5Y cells were cultured in DMEM with $5 \% \mathrm{FBS}$ and $1 \%$ PS. After confluence, at time $=0$, the media was replaced with $50 \%$ horse serum (LOT\# 1671371, GIBCO). Post $2 \mathrm{~h}$ of serum treatment, the high serum media was replaced with serum-free DMEM and collected the cells at $4 \mathrm{~h}$ intervals for protein and total RNA extracts as described in Aurelio Balsalobre procedure [48].

\section{Plasmids constructs and RNA interference}

pCDNA3.1-flag-REGy plasmid was constructed previously [49]. The REGy ShR and ShN plasmids for stable knockdown of REGy were previously described [28]. Full-length BMAL1 plasmid was constructed based on the sequence of human BMAL1 gene. A primer pair was designed to amplify the complete coding region of human BMAL1 gene and conducted PCR amplification in a final reaction volume of $25 \mu \mathrm{l}$, with $2 \mu \mathrm{l}$ of cDNA from $293 \mathrm{~T}$ cells, $2 \mu \mathrm{l}$ of primer mix $(10 \mu \mathrm{M})$, and $12.5 \mu \mathrm{l}$ of Premix Taq, then finally cloned into pcDNA3.1-HA vector.

siRNA targeted for Bmal1 (F-5'-CCACCAACCCAUACACAGAAGCAAA-3') and GSK-3 $\beta$ (F-5'-GCUCCAGAUCAUGAGAAAGCUAGAU-3') were synthesized by Genepharma. SY5Y ShN/ShR cells were seeded in six-well plates and then transfected with the indicated amounts of siRNA with a Lipofectamine RNAiMAX Transfection Reagent Kit (Invitrogen,13778-075). Post $6 \mathrm{~h}$ of the siRNA transfection, the media was changed with DMEM containing $10 \% \mathrm{FBS}$, in the absence of P/S. The cells were incubated for $36 \mathrm{~h}$, and the silencing efficiency was evaluated by western blotting and PCR analysis.

\section{Antibodies}

Given are the primary and secondary antibodies used in western blot analysis with dilution ratios respectively. Anti-Period2-rabbit 1:500 (Abcam, ab180655), anti-BMAL1-rabbit 1:500 (Proteintech, 14268-1-AP), anti-REGY-rabbit 1:250 (Invitrogen, 38-3900), anti- $\beta$-actin-mouse 1:1000 (Sigma, A5316), anti-flagrabbit 1:1000 (MBL life science, M185-3L), and anti-HA-mouse 1:1000 (Abcam, ab130275). The fluorescent-labeled secondary antibodies, anti-Rabbit 1:5000 (IR800) specific to PER2, BMAL1, REG $\gamma$ and Flag antibodies and anti-Mouse 1:10000 (M680) specific to $\beta$-actin and HA antibodies, were purchased from Jackson ImmunoResearch Laboratories, INC.

\section{Western blot analysis}

Total protein from cultured cells and brain tissues were collected in cold RIPA lysis buffer having $(50 \mathrm{mM}$ Tris- $\mathrm{HCl} \mathrm{pH} 7.5,150 \mathrm{mM} \mathrm{NaCl}, 1 \%$ sodium deoxycholate, $1 \%$ Triton X-100, $0.1 \%$ sodium dodecyl sulfate (SDS), $5 \mathrm{mM}$ EDTA, $1 \mathrm{mM}$ Na3VO4, 5-10 mM NaF, $1 \mathrm{mM}$ phenylmethanesulfonylfluoride (PMSF), and protease inhibitor cocktail (Roche)). Total protein concentrations were determined by the BCA Assay Kit (Green-to-purple, Beyotime, China) at $562 \mathrm{~nm}$ absorbance. Equal amounts of whole proteins $(20 \mu \mathrm{g})$ were separated by $10 \%$ SDS-polyacrylamide gel electrophoresis (PAGE) gel along with molecular weight protein marker (PageRuler ${ }^{\mathrm{TM}}$ Prestained Protein Ladder, 10-180 kDa, ThermoFisher Scientific) and transferred the protein from the gel onto nitrocellulose membrane (Millipore, MA, USA). The membrane was blocked with $5 \%$ bovine serum albumin and then immunoblotted with primary antibodies at $4{ }^{\circ} \mathrm{C}$ overnight. After washing with PBST, the membrane was incubated with secondary fluorescent antibodies for $1 \mathrm{~h}$ and the specific signals of the immune-positive protein bands were observed by a fluorescent western blot infrared imaging system LI-COR Odyssey.

\section{Immunoprecipitation}

HEK 293T cells were transiently transfected with specific amounts of pCDNA3.1-Flag-REGY and pcDNA3.1-HA-BMAL1 plasmids (adjusted DNA $1000 \mathrm{ng}$ with pcDNA3.1 vector) for $48 \mathrm{~h}$. The cells were washed with icecold phosphate-buffered saline, collected in ice-cold EP tubes, and harvested for $40 \mathrm{~min}$ in IP lysis buffer $(50 \mathrm{mM}$ Tris- $\mathrm{HCl}$ pH: 7.5, $1 \mathrm{M}$ EDTA, $150 \mathrm{mM} \mathrm{NaCl}, 10 \%$ Glycerol, 1\% Nonidet P-40, protease inhibitor Cocktail, and PMSF). The lysates were incubated with HA or Flag beads (SigmaAldrich, Buchs, Switzerland) at $4{ }^{\circ} \mathrm{C}$ overnight. Washing procedures were applied on the complexes, denatured with $5 \times$ SDS protein loading buffer, and then finally boiled at $100^{\circ} \mathrm{C}$ temperature. The samples were run on $10 \%$ SDS-PAGE gel to identify the protein-protein interactions.

\section{In vitro degradation assay}

For in vitro degradation assay, REG $\gamma$ protein was extracted and purified as described [23]. BMAL1 protein was translated using $50 \mu \mathrm{l}$ in vitro translation system (TNT $40 \mu \mathrm{l}$, Milli-Q H2O $7 \mu \mathrm{l}$, Methionine $1 \mu \mathrm{l}$ ), then kept on $30^{\circ} \mathrm{C}$ for $90 \mathrm{~min}$. Next, the in vitro proteolysis of BMAL1 was carried out by incubating $5 \mu \mathrm{l}$ BMAL1 substrate with $0.25 \mu \mathrm{g}$ of $20 \mathrm{~S}$ proteasome (Boston Biochem) and $2 \mu \mathrm{g}$ of purified REG $\mathrm{in}$ in vitro degradation buffer (10 mM Tris- $\mathrm{HCl} \mathrm{pH}: 7.5,10 \mathrm{mM} \mathrm{KCl}, 10 \%$ glycerol) for $3-5 \mathrm{~h}$ in $25 \mu \mathrm{l}$ reaction volume at $30^{\circ} \mathrm{C}$ with suitable measures. The aliquots of the reaction were finally utilized for western blot analysis.

\section{Gel base and real-time qPCR}

Total RNA was extracted from cells and brain SCN sections in RNA isolation reagent Trizol (Takara). cDNA was synthesized using $2 \mu \mathrm{g}$ of whole-cell RNA in a total $20 \mu$ reaction system with $5 \times$ RT SuperMix (Vazyme, China) and RNase-free $d \mathrm{dH}_{2} \mathrm{O}$. The reverse transcribed RNA was then used for gel base and real-time $\mathrm{qPCR}$ analysis.

For gel base analysis, the following reaction mix in final volume of $20 \mu \mathrm{l}$ was used: forward and reverse primer mix $2 \mu \mathrm{l}, 10 \times$ Taq Buffer $\left(\mathrm{Mg}^{2+}\right.$ plus) $10 \mu \mathrm{l}$, cDNA template $2 \mu \mathrm{l}$, and $\mathrm{mQ}$ water $6 \mu \mathrm{l}$. And then run on PCR instrument with the following program: step $195^{\circ} \mathrm{C} 5 \mathrm{~min}$, step2 for 30 cycles, $\left(95^{\circ} \mathrm{C} 30 \mathrm{~s}, 58^{\circ} \mathrm{C} 30 \mathrm{~s}, 72^{\circ} \mathrm{C} 45 \mathrm{~s}\right)$, step $72^{\circ} \mathrm{C} 10 \mathrm{~min}$, step4 $16^{\circ} \mathrm{C} \sim$

For real-time $\mathrm{QPCR}$ analysis, aliquots of the CDNA were prepared in total volume of $20 \mu \mathrm{l}$ reaction including $10 \mu \mathrm{l} \mathrm{SYBR}$ Green (Vazyme, China), $0.8 \mu \mathrm{l}$ of primers mix, $0.8 \mu \mathrm{l} \mathrm{CDNA}$, and $8.4 \mu \mathrm{lmQ}$ water and then executed on Quantstudio PCR using the following procedure: step $1,95^{\circ} \mathrm{C}$ for $10 \mathrm{~min}$ followed by step 240 cycles at $95^{\circ} \mathrm{C}$ for $30 \mathrm{~s}, 58^{\circ} \mathrm{C}$ for $30 \mathrm{~s}, 72$ for $45 \mathrm{~s}$, and step $3,72^{\circ} \mathrm{C}$ for $10 \mathrm{~min}$. The gene-specific primers for mRNA analysis are given in Supplementary Table 1 .

\section{Cycloheximide assay and doxycycline}

For cycloheximide assay, primary MEF WT/KO and SY5Y ShN/ShR cells were treated with $10 \mu \mathrm{l}$ cycloheximide in DMEM having $10 \%$ FBS and $1 \%$ PS at the indicated time points and then collected the cells for western blot analysis. REG $\gamma$-inducible 293 cells were treated with doxycycline to induce REGY followed by cycloheximide assay.

\section{Statistical analysis}

Statistical analyses were performed using GraphPad Version 6.0, Prism Software Inc., San Diego, CA. Med Associates Running Wheel Data Analysis Software was used to compare the circadian time periods. All the data were expressed as mean \pm standard deviation (SD). Differences between two groups or more were analyzed using Student's $t$ test. $p$ values of $<0.05$ $\left(p<0.05^{*}\right)$ were considered to be of statistical significance.

\section{DATA AVAILABILITY}

Data are available on request from the authors.

\section{REFERENCES}

1. Aschoff J. Circadian rhythms in man. Science 1965;148:1427-32.

2. Welsh DK, Takahashi JS, Kay SA. Suprachiasmatic nucleus: cell autonomy and network properties. Annu Rev Physiol. 2010;72:551-77. 
3. Astiz $M$, Heyde I, Oster H. Mechanisms of communication in the mammalian circadian timing system. Int J Mol Sci. 2019;20:343.

4. Heyde I, Oster H. Differentiating external zeitgeber impact on peripheral circadian clock resetting. Sci Rep. 2019;9:20114.

5. Albrecht U, Sun ZS, Eichele G, Lee CC. A differential response of two putative mammalian circadian regulators, mper1 and mper2, to light. Cell. 1997;91:1055-64.

6. Takahashi JS, Hong HK, Ko CH, McDearmon EL. The genetics of mammalian circadian order and disorder: implications for physiology and disease. Nat Rev Genet. 2008;9:764-75.

7. Sahar S, Sassone-Corsi P. Metabolism and cancer: the circadian clock connection. Nat Rev Cancer. 2009;9:886-96.

8. Bromham L, Penny D. The modern molecular clock. Nat Rev Genet. 2003;4:216-24.

9. Asher G, Schibler U. Crosstalk between components of circadian and metabolic cycles in mammals. Cell Metab. 2011;13:125-37.

10. Reppert SM, Weaver DR. Coordination of circadian timing in mammals. Nature. 2002;418:935-41.

11. Preitner N, Damiola F, Lopez-Molina L, Zakany J, Duboule D, Albrecht U, et al. The orphan nuclear receptor REV-ERBalpha controls circadian transcription within the positive limb of the mammalian circadian oscillator. Cell. 2002;110:251-60.

12. Hirayama J, Sahar S, Grimaldi B, Tamaru T, Takamatsu K, Nakahata Y, et al. CLOCKmediated acetylation of BMAL1 controls circadian function. Nature. 2007;450:1086-90.

13. Sahar S, Zocchi L, Kinoshita C, Borrelli E, Sassone-Corsi P. Regulation of BMAL1 protein stability and circadian function by GSK3beta-mediated phosphorylation. PLoS ONE. 2010;5:e8561.

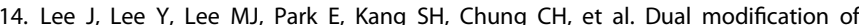
BMAL1 by SUMO2/3 and ubiquitin promotes circadian activation of the CLOCK/ BMAL1 complex. Mol Cell Biol. 2008;28:6056-65.

15. Kwon I, Lee J, Chang SH, Jung NC, Lee BJ, Son GH, et al. BMAL1 shuttling controls transactivation and degradation of the CLOCK/BMAL1 heterodimer. Mol Cell Biol. 2006;26:7318-30.

16. Park N, Kim HD, Cheon S, Row H, Lee J, Han DH, et al. A novel Bmal1 mutant mouse reveals essential roles of the C-terminal domain on circadian rhythms. PLoS ONE. 2015;10:e0138661.

17. Rudic RD, McNamara $\mathrm{P}$, Curtis AM, Boston RC, Panda S, Hogenesch JB, et al BMAL1 and CLOCK, two essential components of the circadian clock, are involved in glucose homeostasis. PLoS Biol. 2004;2:e377.

18. Shi SQ, Ansari TS, McGuinness OP, Wasserman DH, Johnson $\mathrm{CH}$. Circadian disruption leads to insulin resistance and obesity. Curr Biol. 2013;23:372-81.

19. Kondratov RV, Kondratova AA, Gorbacheva VY, Vykhovanets OV, Antoch MP. Early aging and age-related pathologies in mice deficient in BMAL1, the core componentof the circadian clock. Genes Dev. 2006;20:1868-73.

20. Zhou X, Yu R, Long Y, Zhao J, Yu S, Tang Q, et al. BMAL1 deficiency promotes skeletal mandibular hypoplasia via OPG downregulation. Cell Prolif. 2018;51:e12470.

21. Korkmaz T, Aygenli F, Emisoglu H, Ozcelik G, Canturk A, Yilmaz S, et al. Opposite carcinogenic effects of circadian clock gene BMAL1. Sci Rep. 2018;8:16023.

22. Nikaido T, Shimada K, Shibata M, Hata M, Sakamoto M, Takasaki Y, et al. Cloning and nucleotide sequence of cDNA for Ki antigen, a highly conserved nuclear protein detected with sera from patients with systemic lupus erythematosus. Clin Exp Immunol. 1990;79:209-14.

23. Li X, Lonard DM, Jung SY, Malovannaya A, Feng Q, Qin J, et al. The SRC-3/AIB1 coactivator is degraded in a ubiquitin- and ATP-independent manner by the REGgamma proteasome. Cell. 2006;124:381-92.

24. Li X, Amazit L, Long W, Lonard DM, Monaco JJ, O'Malley BW. Ubiquitin- and ATPindependent proteolytic turnover of p21 by the REGgamma-proteasome pathway. Mol Cell. 2007;26:831-42.

25. Lecker SH, Goldberg AL, Mitch WE. Protein degradation by the ubiquitin-proteasome pathway in normal and disease states. J Am Soc Nephrol. 2006;17:1807-19.

26. Wang X, Tu S, Tan J, Tian T, Ran L, Rodier JF, et al. REG gamma: a potential marker in breast cancer and effect on cell cycle and proliferation of breast cancer cell. Med Oncol. 2011;28:31-41.

27. Altman BJ, Hsieh AL, Sengupta A, Krishnanaiah SY, Stine ZE, Walton ZE, et al. MYC disrupts the circadian clock and metabolism in cancer cells. Cell Metab. 2015;22:1009-19.

28. Chen S, Wang Q, Wang L, Chen H, Gao X, Gong D, et al. REGgamma deficiency suppresses tumor progression via stabilizing CK1epsilon in renal cell carcinoma. Cell Death Dis. 2018;9:627.

29. litaka C, Miyazaki K, Akaike T, Ishida N. A role for glycogen synthase kinase-3beta in the mammalian circadian clock. J Biol Chem. 2005;280:29397-402.

30. Hirota T, Lewis WG, Liu AC, Lee JW, Schultz PG, Kay SA. A chemical biology approach reveals period shortening of the mammalian circadian clock by specific inhibition of GSK-3beta. Proc Natl Acad Sci USA. 2008;105:20746-51.

31. Peirson S, Foster RG. Melanopsin: another way of signaling light. Neuron. 2006;49:331-9.
32. Valentinuzzi VS, Scarbrough K, Takahashi JS, Turek FW. Effects of aging on the circadian rhythm of wheel-running activity in C57BL/6 mice. Am J Physiol. 1997;273:R1957-64.

33. Herzog ED, Hermanstyne T, Smyllie NJ, Hastings MH. Regulating the suprachiasmatic nucleus (SCN) circadian clockwork: interplay between cell-autonomous and circuitlevel mechanisms. Cold Spring Harb Perspect Biol. 2017;9:a027706.

34. Miki T, Xu Z, Chen-Goodspeed M, Liu M, Van Oort-Jansen A, Rea MA, et al. PML regulates PER2 nuclear localization and circadian function. EMBO J. 2012;31:1427-39.

35. Mao I, Liu J, Li X, Luo H. REGgamma, a proteasome activator and beyond? Cell Mol Life Sci. 2008;65:3971-80.

36. Sato TK, Panda S, Miraglia LJ, Reyes TM, Rudic RD, McNamara $P$, et al. A functional genomics strategy reveals Rora as a component of the mammalian circadian clock. Neuron. 2004:43:527-37.

37. Low-Zeddies SS, Takahashi JS. Chimera analysis of the Clock mutation in mice shows that complex cellular integration determines circadian behavior. Cell. 2001;105:25-42.

38. Chang HC, Guarente L. SIRT1 mediates central circadian control in the SCN by a mechanism that decays with aging. Cell. 2013;153:1448-60.

39. Masri S, Sassone-Corsi P. The emerging link between cancer, metabolism, and circadian rhythms. Nat Med. 2018;24:1795-803.

40. Liu Z, Selby CP, Yang Y, Lindsey-Boltz LA, Cao X, Eynullazada K, et al. Circadian regulation of c-MYC in mice. Proc Natl Acad Sci USA. 2020;117:21609-17.

41. Gotoh T, Vila-Caballer M, Santos CS, Liu J, Yang J, Finkielstein CV. The circadian factor Period 2 modulates p53 stability and transcriptional activity in unstressed cells. Mol Biol Cell. 2014;25:3081-93.

42. Miki T, Matsumoto $T$, Zhao Z, Lee CC. p53 regulates Period 2 expression and the circadian clock. Nat Commun. 2013;4:2444.

43. He J, Cui L, Zeng Y, Wang G, Zhou P, Yang Y, et al. REGgamma is associated with multiple oncogenic pathways in human cancers. BMC Cancer. 2012;12:75.

44. Yumimoto K, Muneoka T, Tsuboi T, Nakayama KI. Substrate binding promotes formation of the Skp1-Cul1-Fbxl3 (SCF(Fbxl3)) protein complex. J Biol Chem. 2013;288:32766-76

45. Chen S, Yang J, Zhang Y, Duan C, Liu Q, Huang Z, et al. Ubiquitin-conjugating enzyme UBE2O regulates cellular clock function by promoting the degradation of the transcription factor BMAL1. J Biol Chem. 2018;293:11296-309.

46. Barton LF, Runnels HA, Schell TD, Cho Y, Gibbons R, Tevethia SS, et al. Immune defects in $28-\mathrm{kDa}$ proteasome activator gamma-deficient mice. J Immunol. 2004;172:3948-54.

47. Gao X, Wang Q, Wang Y, Liu J, Liu S, Liu J, et al. Author Correction: The REGgamma inhibitor NIP30 increases sensitivity to chemotherapy in p53deficient tumor cells. Nat Commun. 2020;11:4888.

48. Balsalobre A, Damiola F, Schibler U. A serum shock induces circadian gene expression in mammalian tissue culture cells. Cell. 1998;93:929-37.

49. Li L, Zhao D, Wei H, Yao L, Dang Y, Amjad A, et al. REGgamma deficiency promotes premature aging via the casein kinase 1 pathway. Proc Natl Acad Sci USA 2013;110:11005-10.

\section{ACKNOWLEDGEMENTS}

We thank the ECNU Multifunctional Platform for Innovation (011) for keeping and raising mice.

\section{AUTHOR CONTRIBUTIONS}

$\mathrm{XL}$ and $\mathrm{BZ}$ designed research. SK and $\mathrm{HZ}$ performed the molecular, cell biology, and animal experiments. TW and LP were involved in the molecular and cell biology study. YS contributed to the animal behavior experiment. $X G, L L$, and NZ contributed to data analysis. SK, XTL, BZ, and JJF wrote the paper.

\section{FUNDING}

This work was supported in part by grants from the National Natural Science Foundation of China $(82073483,31730017,81672883,82073263,81261120555,31200878,31071875$ 81271742, and 31401012), Shanghai Natural Science Foundation (19JC1411900, 20s11901500, 17ZR1407900), and Shanghai Sailing Program (21YF1431700).

\section{COMPETING INTERESTS}

The authors declare no competing interests.

\section{ETHICS APPROVAL AND CONSENT TO PARTICIPATE}

All animal experiments complied with the National Institutes of Health guide for the care and use of laboratory animals. All studies were reviewed and approved by the Ethics Committee of East China Normal University. 


\section{ADDITIONAL INFORMATION}

Supplementary information The online version contains supplementary material available at https://doi.org/10.1038/s41420-021-00704-9.

Correspondence and requests for materials should be addressed to Nanzhe Zhong, Junjiang Fu, Bianhong Zhang or Xiaotao Li.

Reprints and permission information is available at http://www.nature.com/ reprints

Publisher's note Springer Nature remains neutral with regard to jurisdictional claims in published maps and institutional affiliations.
Open Access This article is licensed under a Creative Commons Attribution 4.0 International License, which permits use, sharing, adaptation, distribution and reproduction in any medium or format, as long as you give appropriate credit to the original author(s) and the source, provide a link to the Creative Commons license, and indicate if changes were made. The images or other third party material in this article are included in the article's Creative Commons license, unless indicated otherwise in a credit line to the material. If material is not included in the article's Creative Commons license and your intended use is not permitted by statutory regulation or exceeds the permitted use, you will need to obtain permission directly from the copyright holder. To view a copy of this license, visit http://creativecommons. org/licenses/by/4.0/.

(c) The Author(s) 2021 